Research Paper

\title{
KIT performed as a driver gene candidate affecting the survival status of patients with stomach adenocarcinoma
}

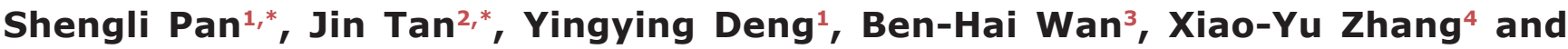 \\ Bu-Gao Guan ${ }^{3}$ \\ ${ }^{1}$ Shanghai Eighth People's Hospital, Shanghai, China \\ ${ }^{2}$ Department of Thoracic Surgery, Huai'an First People's Hospital, Nanjing Medical University, Huai'an, China \\ ${ }^{3}$ Department of General Surgery, People's Hospital of Jinhu, Huai'an, China \\ ${ }^{4}$ Division of Gastrointestinal Surgery, Department of General Surgery, The Affiliated Huai'an Hospital of Xuzhou Medical \\ University and The Second People's Hospital of Huai'an, Huai'an, China \\ * Co-first authors \\ Correspondence to: Bu-Gao Guan, email: 2531257743@qq.com \\ Xiao-Yu Zhang, email: zhangxiaoyu_zx@163.com
}

Keywords: stomach adenocarcinoma, prognosis, TCGA, KIT, driver gene candidates

Received: March 12, $2017 \quad$ Accepted: June 04, $2017 \quad$ Published: July 26, 2017

Copyright: Pan et al. This is an open-access article distributed under the terms of the Creative Commons Attribution License 3.0 (CC BY 3.0), which permits unrestricted use, distribution, and reproduction in any medium, provided the original author and source are credited.

\section{ABSTRACT}

Stomach adenocarcinoma is estimated to cause 10,000 deaths in the US in 2016 and is the third most deadly cancer in China. We aim to identify the proteins and the genes that have impact on the prognosis of patients with stomach adenocarcinoma. Data of patients with stomach adenocarcinoma were retrieved from The Cancer Genome Atlas (TCGA). Proteins whose expression levels were highly correlated with survival status of patients were figured out. The expression levels of their mRNAs and their roles in the pathway were used to determine the driver gene candidates. The effects of mutations on the genes encoding KIT on mRNA expressions were carried. Ten antibodies were figured out to have significant correlation with stomach cancer prognosis. The coefficients of COXPH models matches their roles in the previous studies. The expression levels of mRNAs versus proteins suggested that KIT might act as a driver gene, which was also the central in the pathway of other selected proteins. The missense mutations on the gene encoding KIT led to the low expression of its mRNAs and there were much fewer nonsense mutations compared with other genes. It suggested that the important role of KIT as an oncogene in the progression of cancer, as well as a tyrosine-protein kinase during the normal activity. Ten antibodies, corresponding to fifteen proteins, were highly correlated with patients' survival time, within which KIT played a critical roles. It suggested that KIT might be used as biomarker or as target of cancer therapies.

\section{INTRODUCTION}

It was estimated that there would be over 26,000 new stomach cancer cases and over 10,000 deaths caused by it in the United States in 2016 [1]. In China, stomach adenocarcinoma is the third most prevalent cancer, as well as the third most deadly [2]. The 5-Year relative survival rate of stomach adenocarcinoma increased from $20 \%$ before 1990 to $30 \%$ at present compared with any average survival rate of $69 \%$ for all kinds of cancer [1]. It prevalence and mortality urged us to find out the factors affecting its incidence and prognosis.

The direct molecules regulating the body status are proteins instead of DNA or RNA. However, most of cancer studies at present focused on mRNA expression levels, mutations or even miRNA expression levels, but few studies used large-scale protein expression levels considering the difficulties to obtain such information. Luckily, M.D. Anderson Reverse Phase Protein Array Core provided the expression levels proteins which are targeted 
by 218 different antibodies on TCGA. Such data can help us to study the cancer further. On the meantime, there are various differences, from mutations to mRNA expression levels, from copy number variation to protein translation, between cancer patients and normal populations. However, most of differences were passengers which were neutral or influenced by other aberrant behaviors. Driver genes, which are responsible for cancer development through specific alterations, were hard to be figured out unfortunately [3].

In this study, we started from protein expression levels to find out the proteins that were significantly correlated with survival time of patients. Afterward, mRNA expression levels and somatic mutations information will be utilized to identify the driver gene candidates. The final results suggested that KIT, as well as the genes encoding these proteins, might play a critical and central role in the survival status of stomach patients.

\section{RESULTS}

\section{Proteins correlated with survival status}

There were totally 218 antibodies used to detect protein expression levels, though some antibodies unavailable for a certain group of patients. The overall expression patterns for these antibodies were plotted as a heatmap (Figure 1).

COXPH models were built separately for all the antibodies based on 347 patients with protein expressed information. Ten antibodies with a p-value less than 0.05 were extracted. The detailed information for these antibodies was listed in Table 1. Campared with previous studies, we found that most of these genes were tumor suppressors or activators. Their role also corresponded to their coefficients of the COXPH model, except for Src_pY416. Interestingly, Src_pY416 antibody targets six different proteins, which might make it more complex

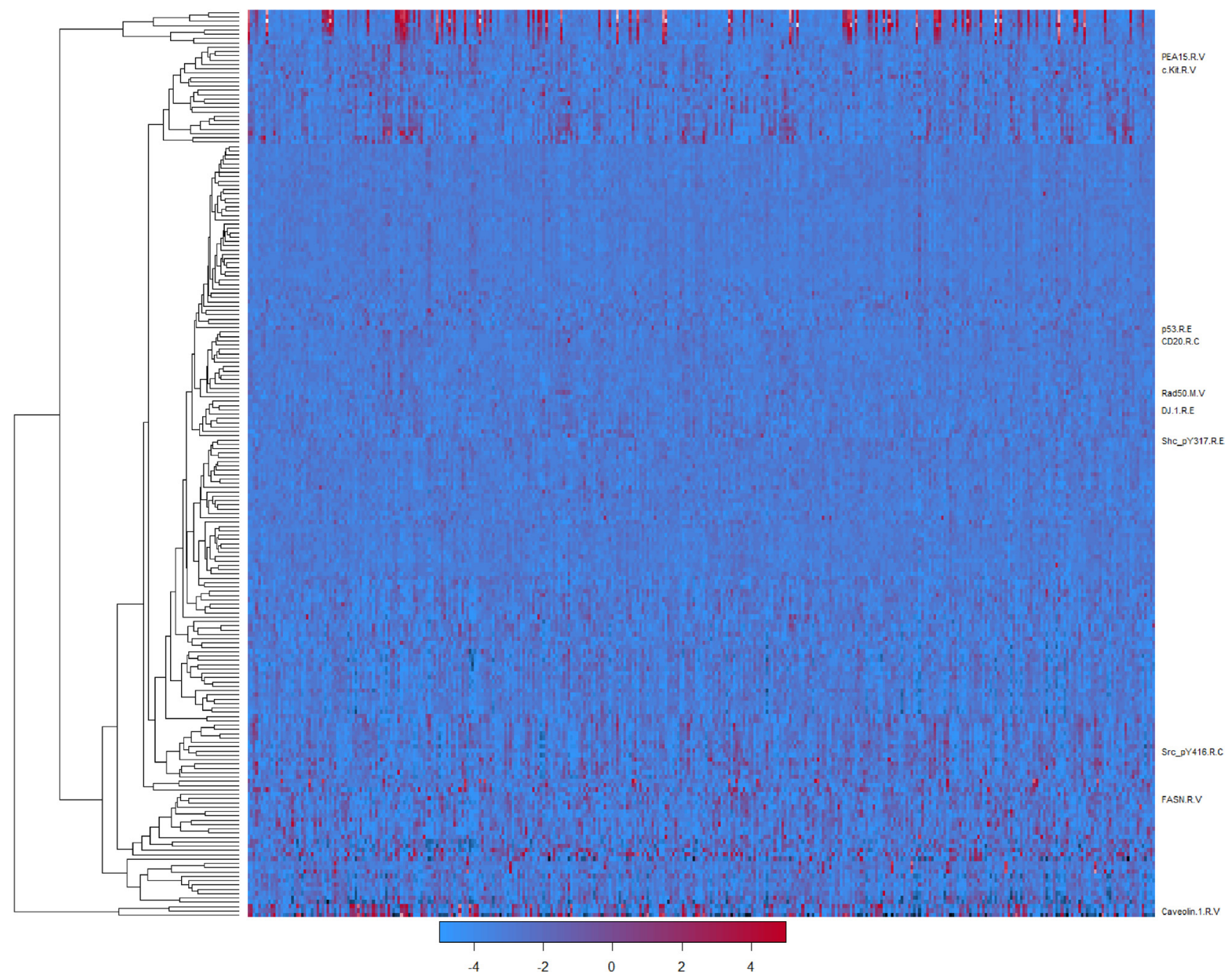

Figure 1: Heatmap for the expression levels of proteins. The rows represented different antibodies and the columns were patients. Red meant the high expression levels and blue meant the opposite. The antibodies having a p-value lower than 0.05 in the COXPH model were annotated on the right. Antibodies that were unavailable in any patients were omitted. 
Table 1: The summary of antibodies that highly correlated with survival status of patients with stomach cancer

\begin{tabular}{lccccc}
\hline Antibody & Protein & coef & exp(coef) & p_value & Note \\
\hline Shc_pY317 & SHC1 & -1.24 & 0.29 & 0.041 & Tumor suppressor \\
p53 & TP53 & -1.08 & 0.34 & 0.017 & Tumor suppressor \\
Src_pY416 & SRC, LYN, FYN, LCK, YES1, HCK & -0.69 & 0.50 & 0.024 & Proto-oncogene \\
FASN & FASN & -0.56 & 0.57 & 0.031 & \\
Caveolin-1 & CAV1 & 0.38 & 1.46 & 0.013 & Tumor suppressor \\
c-Kit & KIT & 0.69 & 2.00 & 0.026 & Tumor activator \\
DJ1 & PARK7 & 1.10 & 3.01 & 0.030 & Tumor activator \\
PEA-15 & PEA15 & 1.23 & 3.44 & 0.012 & Tumor activator \\
Rad50 & RAD50 & 1.62 & 5.03 & 0.001 & Tumor activator \\
CD20 & CD20 & 2.23 & 9.35 & 0.031 & Tumor activator \\
\hline
\end{tabular}

to figure out theirroles on the survival status. We could not determine which protein(s) resulted in the significant results with limited data.

For these 10 antibodies, their expression patterns were not totally the same (Figure 1), though all of them were highly correlated with survival time of patients. This suggested their different roles in the stomach cancer progress.

The Kaplan-Meier survival curves of four proteins were plotted as examples in Figure 2. It indicated that some of these proteins were able to separate patients with good prognosis and poor prognosis, though some of them, like CAV1, failed in separating the two groups.

\section{Networks and pathways of survival related proteins}

These proteins were highly correlated with each other. Most of these proteins had intact protein-protein interactions with high confidence and all of them had some certain evidences to suggest that they had physical interactions (Figure 3A). Such results indicated that any aberrant behavior of one protein might affect all of other proteins.

The proteins interacted with each other and most of proteins acted as a hub, including KIT, proteins targeted by Src_pY416, TP53 and so forth. It was difficult to figure out the most important proteins in such network, but when we looked at the pathway they involved, KIT performed as a central part among these proteins. When searching for the pathways involving these proteins on KEGG, it seemed that KIT was the upstream of most of other proteins (Figure 3B). For example, in the RAS signaling pathway, KIT directly activates SHC through protein-protein interaction; KIT is also the most upstream protein in PI3K-Akt and is able to regulate the function of TP53 indirectly; it works with other proteins to convert inactive RAP1 into active status and in further active SRC and CAV1. All of the pathways shared with KIT were listed in Table 2. The pathway information is unavailable or incomplete for some genes, whichwere not included in the table.

\section{Driver proteins versus passengers}

Driver genes for cancer were responsible for the cancer incidence, progression and prognosis. Even though the levels of protein mentioned above seemed highly correlated with patients' survival time, it is difficult to say that these proteins and the genes encoding them caused the phenomenon directly. After all, the levels of proteins were regulated by complex microenvironment including the different ion concentration, other proteins, miRNAs, mRNAs and so forth.

It was reported that only one-thirds of mRNA expression levels were positively related with their protein expression levels with high correlation coefficients [4]. If the mRNA expression levels were significantly correlated with the protein expression, it became a good support that the impact of protein on the patients' survival time might come from the mRNA expression level and in future the DNA methylation and SNPs instead of regulation of other factors. Here only KIT had a significant correlation between mRNA expression levels and protein expression levels with a p-value lower than 2.2e-16 (Figure 4A). Other proteins had a low correlation coefficients and Figure 3B showed two examples The correlation coefficient for KIT was higher than 0.50 , higher than the cutoff used in study of Gry, Rimini [4], which suggested that the relation is robust. Other proteins had poorer correlation.

It seemed that KIT played a central role in the analysis of pathways. And the correlation between mRNA expression levels and protein expression levels also suggested that KIT might be a driven genes instead 
of passenger genes. Also, the poor correlation of other proteins and the tight relation with KIT provided a possibility that KIT regulated the levels of other survivalrelated proteins and worked together to affect the prognosis of stomach cancer.

We also used the mRNA expression levels of these genes to fit the COXPH model but it seemed that they were not good biomarkers for the survival time. Even for the mRNA of KIT, the p-value was close to 0.1 .

\section{Mutations of gene encoding KIT}

We have suggested that the level of KIT mRNA had direct impact on its protein expression levels, and afterwards we would like to study the relationship between the DNA SNPs and mRNA expression levels.

First, most of the mutations on KIT were missense mutations and silent mutations (Figure 5A), unlike other

KIT

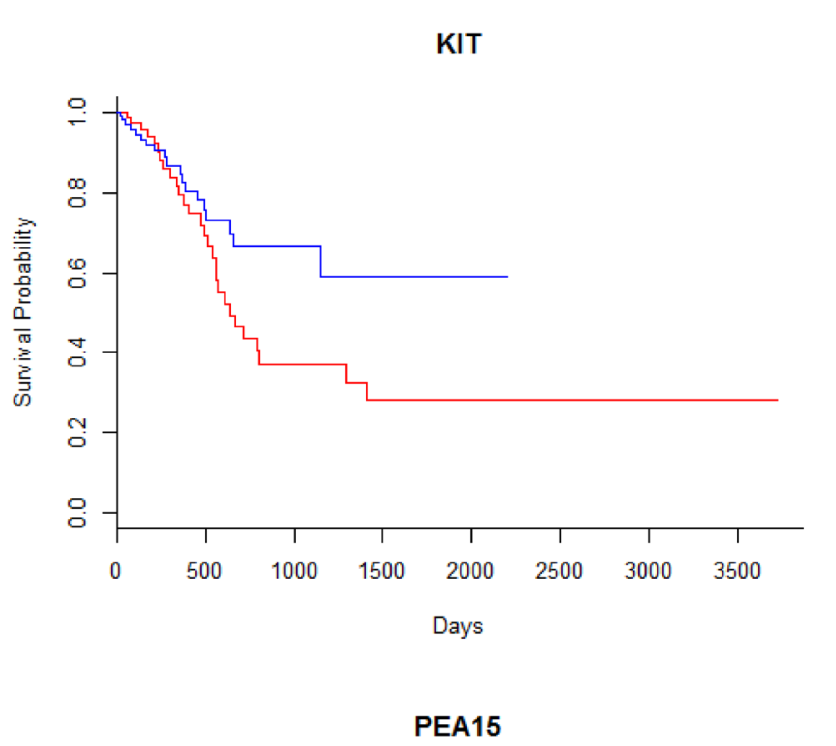

PEA15

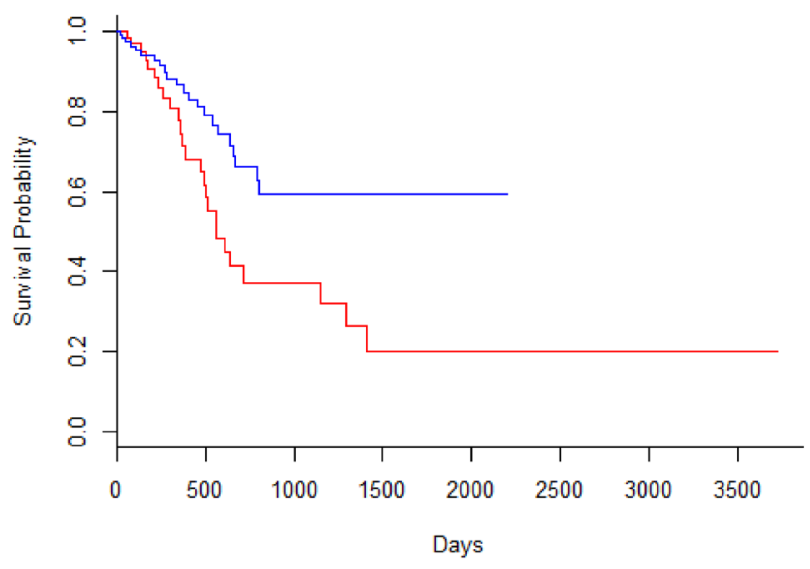

genes which had a lot of nonsense mutations. Second, patients with missense mutations on KIT had significantly lower level of mRNA with a p-value of 0.016 than patients without mutations (Figure 5B). Even for the patients with only silent mutaions, their mRNA levels seemed a little lower than wild-type, though the p-value is insignificant probably caused by the small sample. This hinted that the mutations on KIT might be able to affect the stability or transcription of its mRNA.

The overall mutations were summarized in Figure 6C. To find out the influence of these mutated sites, the cis-regulatory elements on the mRNA were predicted on RegRNA 2.0 (Figure 6A) and the protein structure was predicted using SWISS-MODEL (figure not shown). Due to the size of the protein, the SWISSMODEL only simulated part of the protein and the whole protein structure reported by Roskoski [5] was shown in Figure 6B.
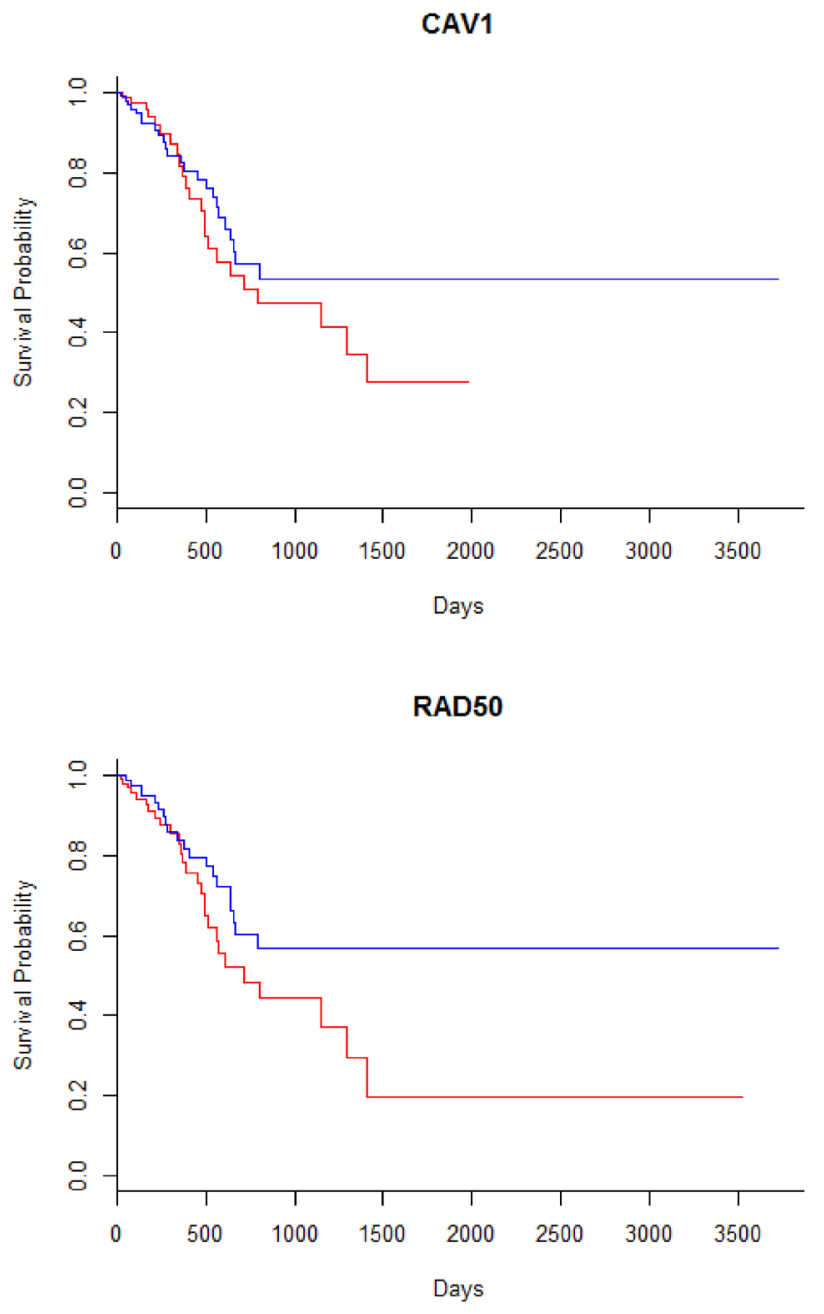

Figure 2: The survival curves of the patients based on the expression levels of the specific proteins. Patients were separately into two even groups according to the hazard rate. The blue lines represented the patients with low hazard rate and the red lines represented high risk. 


\section{DISCUSSION}

\section{Missense mutations on KIT}

KIT is a tyrosine-protein kinase, which performed various functions. It played important role in the process of hematopoiesis, melanogenesis, and gametogenesis [6]. It also regulates cell shape, motility, and adhesion via cytoskeletal changes. KIT is expressed as a glycosylated transmembrane protein with an extracellular domain, a transmembrane region, and a tyrosine kinase domain (Figure 6B). As a result, any nonsense mutations might be lethal and any missense mutations affected the body dramatically. It was reported that the allelic variants of KIT would lead to various diseases including piebaldism [7], mast cell leukemia [8], gastrointestinal stromal tumor [9] and so on.

The missense mutations on genes encoding KIT were summarized in Figure 6C. One intriguing finding is that most of these mutations were not known oncogenic mutations, except for the protein position 560, whose mutations will lead to gastrointestinal stromal tumors and mastocytosis [5]. Also, considering the mRNA expression levels in patients with KIT mutations and those without mutations, these missense mutations may play a more important role on its mRNAs. KIT mRNAs owned various

A

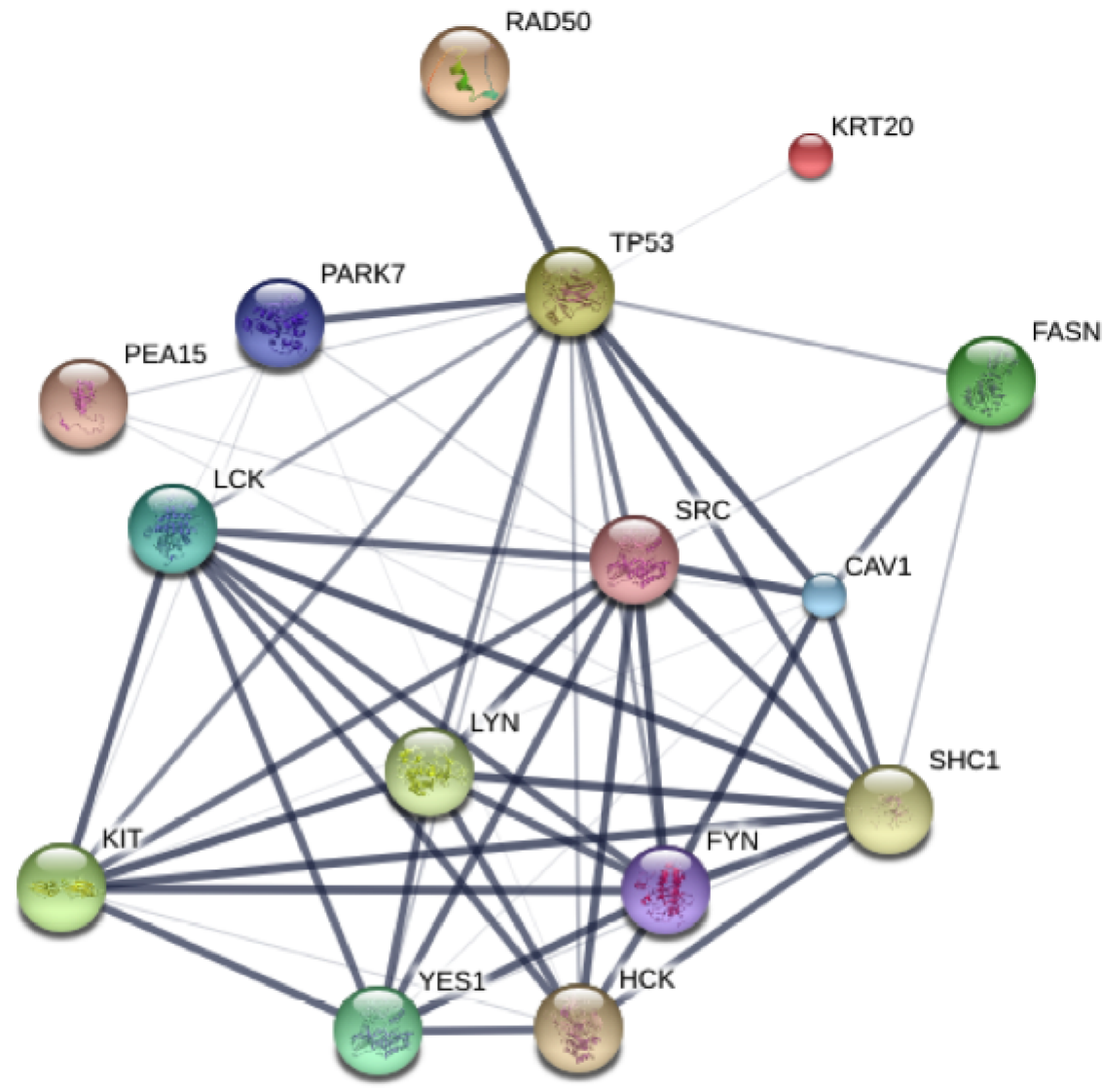

B

RAS signaling pathway

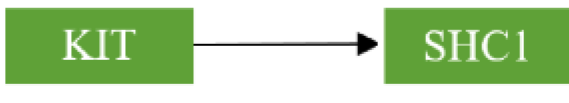

PI3K-Akt signaling pathway

\section{KIT}

PI3K

\section{TP53}

\section{Rap1 signaling pathway and Endocytosis}

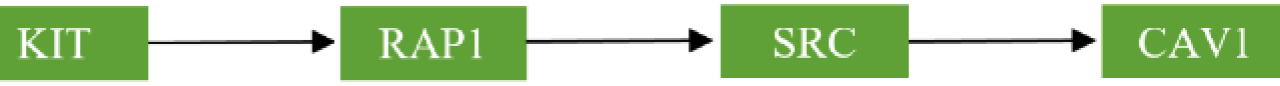

Figure 3: The networks and pathways involvig proteins that were correlated with patients' survival time. (A) The protein-protein interactions between proteins. The confidences of the interactions were annotated by the width of edges. (B) The selected regulated relationship between KIT and other proteins. 
Table 2: The shared pathways with KIT

\begin{tabular}{lll}
\hline Pathway ID & KIT involved pathway & Shared proteins \\
\hline hsa04014 & Ras signaling pathway & SHC1 \\
hsa04015 & Rap1 signaling pathway & SRC \\
hsa04072 & Phospholipase D signaling pathway & SHC1, FYN \\
hsa04144 & Endocytosis & CAV1, SRC \\
hsa04151 & PI3K-Akt signaling pathway & TP53 \\
\hline
\end{tabular}

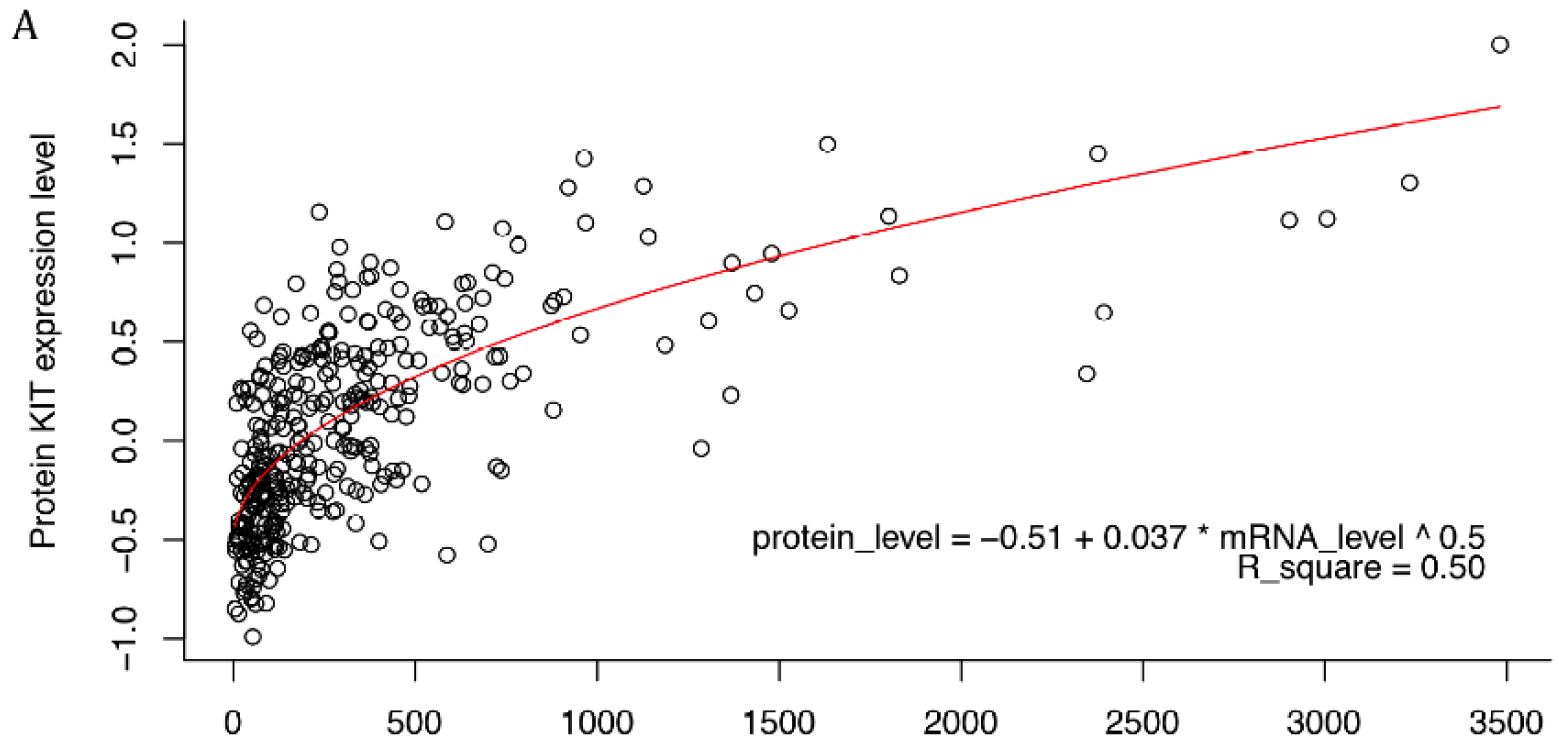

KIT mRNA expression level

$\mathrm{B}$

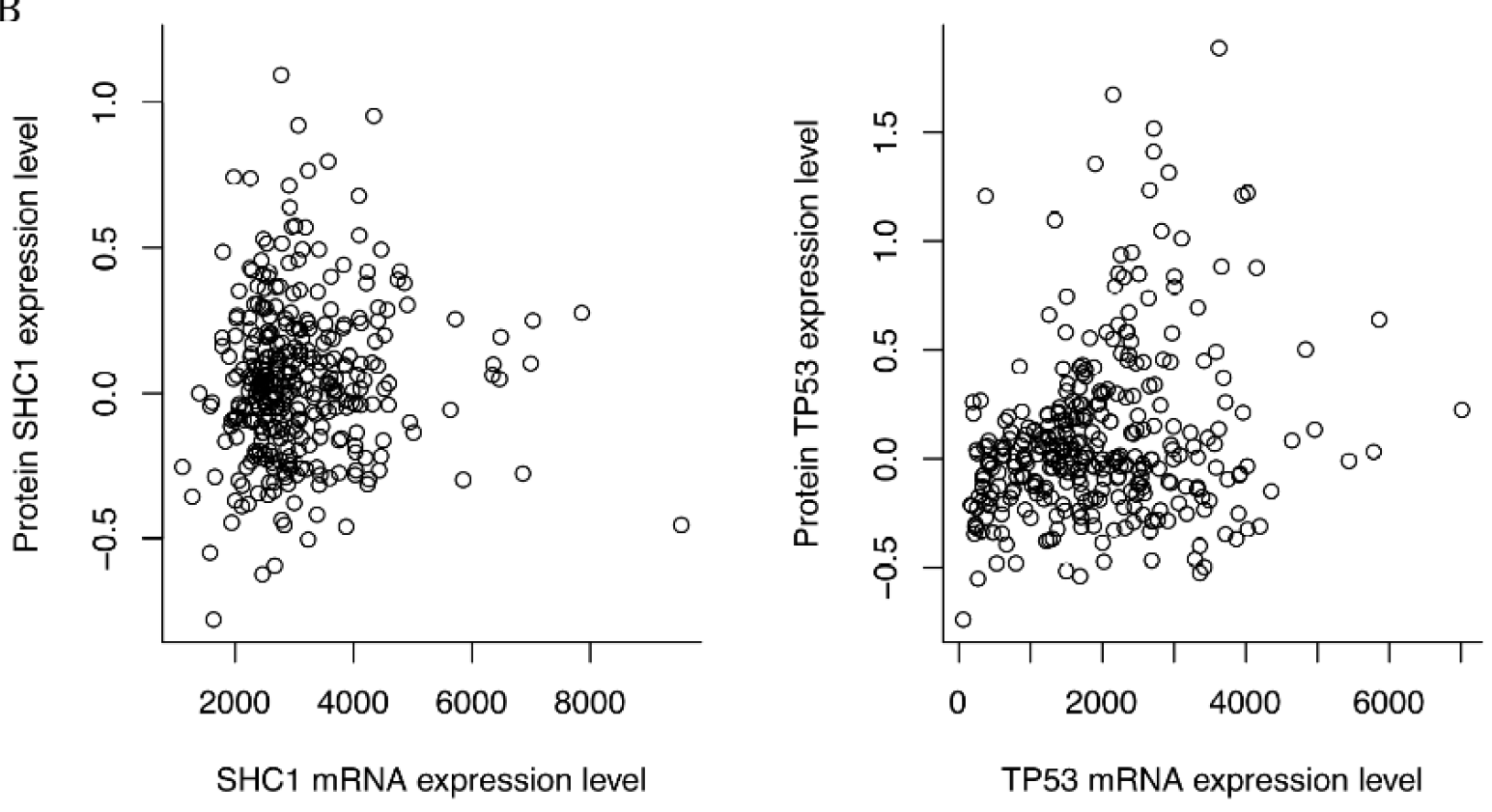

Figure 4: The scatter plot of mRNA expression levels versus corresponding protein expression levels. (A) The plot of KIT. The curve of regression was plot as red which had a R-squared as 0.5. (B) The plot of SHC1 and TP53. Except for KIT, none of survivalrelated proteins had significantly correlation with their mRNA expression levels. Only two examples were shown here. 
A
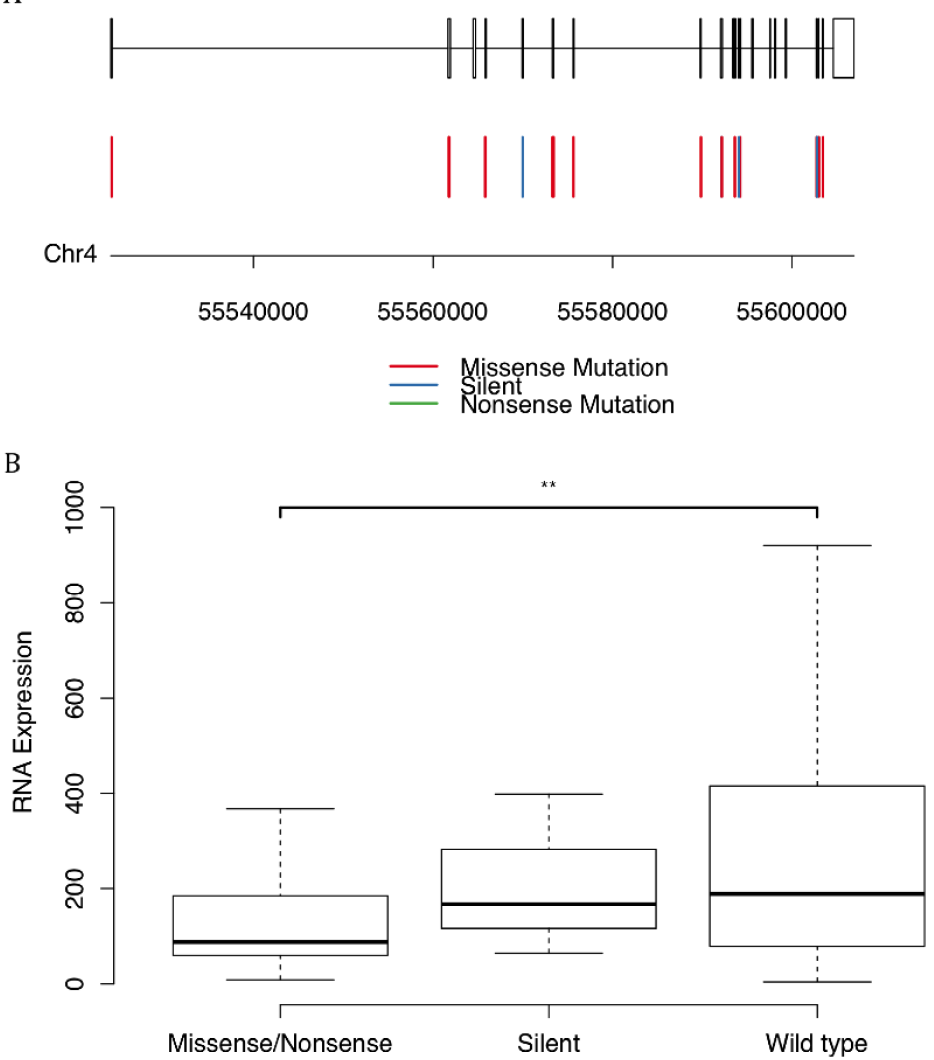

Figure 5: Mutations of the gene encoding protein KIT. (A) The mutation positions and types. (B) The mRNA expression levels versus the status of mutations. The symbol '**' represented a p-value lower than 0.05 .

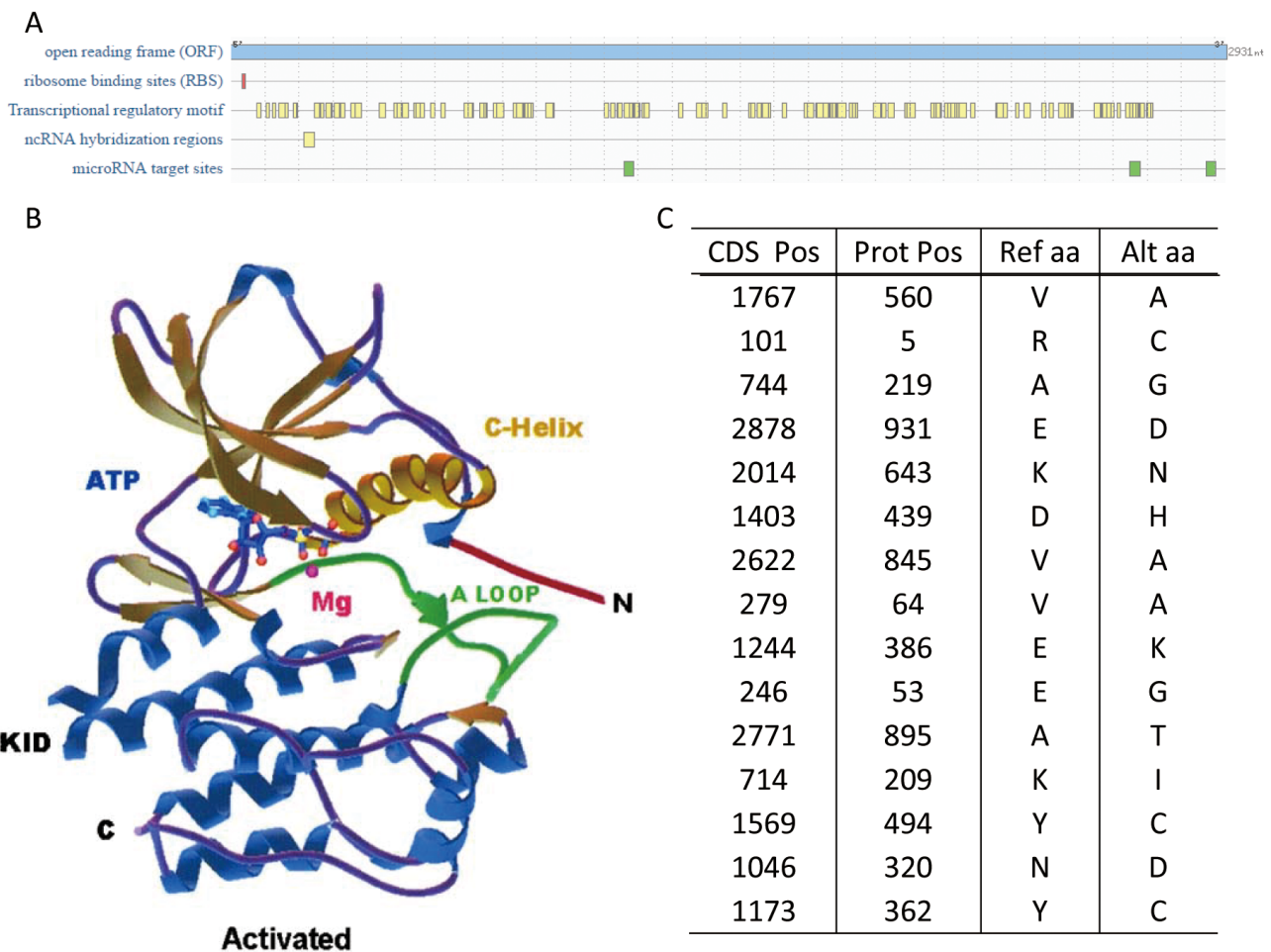

Figure 6: Genomic mutations of KIT. (A) The cis-regulatory elements on KIT mRNA. (B) The protein structure of KIT. This graph came from the paper of Roskoski [5]. (C) The missense mutations in the patients from TCGA. 
cis-regulatory elements (Figure 6A) with functions to stabilize, mobilize and translate KIT.

Another important feature of the mutations on genes encoding KIT is that most of the mutations were missense mutations or silent mutations. We also checked the mutation status of other oncogenes and their mutations were most nonsense mutations. This may suggest the critical function of KIT in vivo except for its contribution for the cancer progression. However, the bench experiments, which engineer the KIT gene by inducing mutations, knockout or duplicating, are needed in further to validate our assumptions.

\section{Utilization of protein expression levels}

We can easily find various cancer related papers talking about mRNA expression levels, but much fewer papers paid the attention on the protein expression levels. The most important reason is that it is quite hard and expensive to obtain the protein expression level. Here, we suggested that utilizing public data from TCGA and other database was a good choice to analyze protein information since these protein information cannot be found in the TCGA data matrix. Also, starting from the protein expression levels, we can directly find the molecules affected our body in a direct way. On the other hand, the protein expression levels were measured through antibody, which could not distinguish similar proteins. For example, antibody Src_pY416 targeted SRC, LYN, FYN, LCK, YES1 and HCK. In this study, we simply assumed that all the targets of Src_pY416 were associated with the patients' survival, though more experiments were needed to identify the exact associated proteins.

\section{MATERIALS AND METHODS}

\section{TCGA stomach cancer}

Clinical information, protein expression levels, mRNA expression levels and somatic mutation information of patients with stomach adenocarcinoma were retrieved from The Cancer Genome Atlas (TCGA). The protein expression levels came from the platform of M.D. Anderson reverse phase protein array. The expression level of mRNA and somatic mutations were obtained under platform of Illumina HiSeq 2000 RNA sequencing version 2 and Illumina DNA sequencing, respectively. This dataset contains 478 patients, within which 347, 443 and 439 patients had protein, mRNA and mutations information.

\section{Survival related proteins}

There were totally 218 antibodies used to detect protein expression levels. With 347 patients having protein expressed information, COXPH models were built separately for all the antibodies [11]. Ten antibodies with a p-value less than 0.05 were extracted. The KaplanMeier survival curves [12] were plotted to visualize the performance by dividing patients into two even groups using hazard rate from COXPH model.

\section{Network and pathways}

The protein-protein interactions among these proteins were plotted using STRING [13]. Every proteins were searched in the database KEGG to find out the pathway they involved and the regulation relationship among them [14].

\section{Identification of driven protein}

The expression levels of mRNA corresponding to the proteins related with survival status of patients with stomach adenocarcinoma were extracted. The relationship between mRNA expression levels versus protein expression levels were shown as scatterplots. Regression was then carried out. KIT was found to have high correlation between mRNA and protein expression levels. The following analysis would focus on this protein and the gene encoding it.

\section{Analysis on KIT}

Mutations of the genes encoding these proteins were available in the platform using Illumina DNA sequencing. The sequences of DNA and also the gene model were retrieved from USCS [15]. The differences of mRNA expression levels between patients with or without KIT mutations were performed using Student's T test and visualized using box-and-whisker plots.

The protein structure of KIT was predicted using SWISS-MODEL [16] and the cis-regulatory elements on the KIT mRNA were analyzed through RegRNA 2.0 [17].

\section{CONCLUSIONS}

In this study, we at first found the proteins highly correlated with survival time of patients with stomach adenocarcinoma. Based on this analysis, KIT was suggested to be a critical and central molecule in the biological process and it performed as a driver gene instead of passenger genes. As a result, it may perform in an aberrant way in early stage that it can be used as a good biomarker. Also the targeted drugs on KIT may be the most critical. There are already targeted therapies targeting KIT, including Axitinib, Cabozantinib, Imatinib, Pazopanib, Sorafenib and so forth [10]. More targeted therapies working on KIT may be found in the future.

\section{CONFLICTS OF INTEREST}

None of the authors have any competing interest. 


\section{ACKNOWLEDGMENTS}

We thank Dr. Ai-Mei Gao from Shanghai General Hospital, Shanghai Jiaotong University and Dr. Zun-Ping Ke from Shanghai Fifth Hospital, Fudan University from their assistance in study design.

\section{REFERENCES}

1. Siegel RL, Miller KD, Jemal A. Cancer statistics, 2016. CA Cancer J Clin. 2016; 66:7-30.

2. Chen W, Zheng R, Zeng H, Zhang S, He J. Annual report on status of cancer in China, 2011. Chin J Cancer Res. 2015; 27:2-12.

3. Schroeder MP, Rubio-Perez C, Tamborero D, GonzalezPerez A, Lopez-Bigas N. OncodriveROLE classifies cancer driver genes in loss of function and activating mode of action. Bioinformatics. 2014; 30:i549-555.

4. Gry M, Rimini R, Stromberg S, Asplund A, Ponten F, Uhlen M, Nilsson P. Correlations between RNA and protein expression profiles in 23 human cell lines. BMC Genomics. 2009; 10:365.

5. Roskoski R Jr. Structure, regulation of Kit protein-tyrosine kinase - the stem cell factor receptor. Biochem Biophys Res Commun. 2005; 338:1307-1315.

6. Rothschild G, Sottas CM, Kissel H, Agosti V, Manova $\mathrm{K}$, Hardy MP, Besmer P. A role for kit receptor signaling in Leydig cell steroidogenesis. Biol Reprod. 2003; 69:925-932.

7. Richards KA, Fukai K, Oiso N, Paller AS. A novel KIT mutation results in piebaldism with progressive depigmentation. J Am Acad Dermatol. 2001; 44:288-292.

8. Furitsu T, Tsujimura T, Tono T, Ikeda H, Kitayama H, Koshimizu U, Sugahara H, Butterfield JH, Ashman LK, Kanayama Y, et al. Identification of mutations in the coding sequence of the proto-oncogene c-kit in a human mast cell leukemia cell line causing ligand-independent activation of c-kit product. J Clin Invest. 1993; 92:1736-1744.

9. Hirota S. Gain-of-function mutations of c-kit in human gastrointestinal stromal tumors. Science. 1998; 279:577-580.

10. Abramson R. (2016). Overview of Targeted Therapies for Cancer.

11. Andersen PK, Gill RD. Cox's regression model for counting processes: a large sample study. Ann Stat. 1982; 10:1100-1120.

12. Goel MK, Khanna P, Kishore J. Understanding survival analysis: Kaplan-Meier estimate. Int J Ayurveda Res. 2010; 1:274-278.

13. Szklarczyk D, Franceschini A, Wyder S, Forslund K, Heller D, Huerta-Cepas J, Simonovic M, Roth A, Santos A, Tsafou KP, Kuhn M, Bork P, Jensen LJ, von Mering C. STRING v10: protein-protein interaction networks, integrated over the tree of life. Nucleic Acids Res. 2015; 43:D447-452.

14. Kanehisa M, Sato Y, Kawashima M, Furumichi M, Tanabe $\mathrm{M}$. KEGG as a reference resource for gene and protein annotation. Nucleic Acids Res. 2016; 44:D457-462.

15. Karolchik D, Hinrichs AS, Furey TS, Roskin KM, Sugnet CW, Haussler D, Kent WJ. The UCSC Table Browser data retrieval tool. Nucleic Acids Res. 2004; 32:D493-496.

16. Biasini M, Bienert S, Waterhouse A, Arnold K, Studer G, Schmidt T, Kiefer F, Gallo Cassarino T, Bertoni M, Bordoli L, Schwede T. SWISS-MODEL: modelling protein tertiary and quaternary structure using evolutionary information. Nucleic Acids Res. 2014; 42:W252-258.

17. Chang TH, Huang HY, Hsu JB, Weng SL, Horng JT, Huang HD. An enhanced computational platform for investigating the roles of regulatory RNA and for identifying functional RNA motifs. BMC Bioinformatics. 2013; 14:S4. 